PROCEEDINGS OF THE

AMERICAN MATHEMATICAL SOCIETY

Volume 140, Number 8, August 2012, Pages 2855-2872

S 0002-9939(2011)11116-X

Article electronically published on December 15, 2011

\title{
ON A NON-ABELIAN POINCARÉ LEMMA
}

\author{
THEODORE TH. VORONOV
}

(Communicated by Varghese Mathai)

Abstract. We show that a well-known result on solutions of the MaurerCartan equation extends to arbitrary (inhomogeneous) odd forms: any such form with values in a Lie superalgebra satisfying $d \omega+\omega^{2}=0$ is gaugeequivalent to a constant,

$$
\omega=g C g^{-1}-d g g^{-1} .
$$

This follows from a non-Abelian version of a chain homotopy formula making use of multiplicative integrals. An application to Lie algebroids and their nonlinear analogs is given.

Constructions presented here generalize to an abstract setting of differential Lie superalgebras where we arrive at the statement that odd elements (not necessarily satisfying the Maurer-Cartan equation) are homotopic - in a certain particular sense - if and only if they are gauge-equivalent.

\section{INTRODUCTION}

It is well known that a 1 -form with values in a Lie algebra $\mathfrak{g}$ satisfying the Maurer-Cartan equation

$$
d \omega+\frac{1}{2}[\omega, \omega]=0
$$

possesses a 'logarithmic primitive' locally or for a simply-connected domain

$$
\omega=-d g g^{-1}
$$

for some $G$-valued function, where $G$ is a Lie group with the Lie algebra $\mathfrak{g}$. Here and in the sequel we write all formulas as if our algebras and groups were matrix; it makes the equations more transparent, and certainly nothing prevents us from rephrasing them in an abstract form. Therefore the main equation (1.1) will also be written as

$$
d \omega+\omega^{2}=0
$$

Received by the editors December 4, 2009 and, in revised form, March 10, 2011.

2010 Mathematics Subject Classification. Primary 53D17; Secondary 18G50, 58A50, 58C50, $58 \mathrm{H} 05$.

Key words and phrases. Maurer-Cartan equation, Lie superalgebras, differential forms, supermanifolds, Lie algebroids, homological vector fields, multiplicative integral, $Q$-manifolds, Quillen's superconnection.

(C)2011 American Mathematical Society Reverts to public domain 28 years from publication 
In the physical parlance, (1.2) means that the $\mathfrak{g}$-valued 1-form $\omega$ is a 'pure gauge'; i.e., the gauge potential $\omega$ is gauge-equivalent to zero. Mathematically (1.1) means that the operation

$$
d+\omega
$$

defines a flat connection and $g=g(x)$ in (1.2) is a choice of a parallel frame by which $d+\omega$ can be reduced to the trivial connection $d$.

On the other hand, in the Abelian case (for example, for scalar-valued forms), the Maurer-Cartan equation simply becomes $d \omega=0$, equation (1.2) becomes $\omega=$ $-d \ln g=d f$ for a non-zero function $g(x)=e^{-f(x)}$, and the whole statement is just a particular case of the Poincaré lemma for 1-forms.

We show that there is a non-Abelian analog of the Poincaré lemma in full generality. Namely, instead of a one-form we consider an arbitrary odd form $\omega$ with values in a Lie superalgebra $\mathfrak{g}$. Here 'odd' means, in the sense of total $\mathbb{Z}_{2}$-grading (parity), taking account of parity of elements of the Lie superalgebra. The form may very well be inhomogeneous w.r.t. degree; i.e., it may be the sum of a 0 -form, a 1-form, a 2-form, etc., or even be a pseudodifferential form on a supermanifold.

The statement then is as follows. If the odd form $\omega$ satisfies (1.1) or (1.3), then it is gauge-equivalent to a constant: there is a $G$-valued form $g$ (necessarily even) such that

$$
\omega=g C g^{-1}-d g g^{-1},
$$

where $C$ is a constant odd element of the superalgebra $\mathfrak{g}$ satisfying $C^{2}=0$. Here $G$ is a Lie supergroup corresponding to the Lie superalgebra $\mathfrak{g}$. (Note that $g$ is not a function as in the classical situation, but a form. How to understand such ' $G$-valued forms' is explained in the main text.)

This is a precise analog of the statement that every closed form is exact plus a constant in a contractible domain. The appearance of a constant $C$ in (1.5) is a crucial non-trivial feature, distinguishing our statement from the well-known case when $\omega$ is a $\mathfrak{g}$-valued 1 -form. The appearance of a constant may seem a very innocent change, at a superficial glance, but examples show that it is not. (In fact, this odd $\mathfrak{g}$-valued constant may be a whole homological vector field defining a particular structure; see section 4.)

From a 'connections viewpoint', the meaning of our statement is that the operation (1.4), which now can be interpreted as, say, Quillen's superconnection [9] (it is not an ordinary connection if $\omega$ is not a 1-form), is equivalent, by taking the conjugation with a group element $g$ (an invertible even form taking values in $G$ ), to

$$
d+C .
$$

We discuss an application to Lie algebroids and their non-linear analogs. No doubt, the statement has other applications.

Our 'non-Abelian Poincaré lemma' is deduced from a more general theorem, which may be seen as the non-Abelian analog of a homotopy formula for differential forms. In the last section we discuss a relation with homotopy theory in a more abstract setup of differential Lie superalgebras and differential Lie supergroups.

\section{2. 'Multiplicative Direct image' and A HOMOtopy formula}

Consider a (super)manifold $M$ and the direct product $M \times I$ where $I=[0,1]$. Let $\mathfrak{g}$ be a Lie superalgebra with a Lie supergroup $G$. 
Remark 2.1. The Lie superalgebra $\mathfrak{g}$ may be finite-dimensional, but not necessarily. Of course, for infinite-dimensional algebras, interesting for applications, the existence of a corresponding supergroup $G$ and of multiplicative integrals (see below) need to be established in each concrete case. One particular class of examples to which considerations of this paper readily apply consists of groups (or supergroups) of transformations of a supermanifold. As mentioned above, in the sequel we use the notation mimicking the case of matrix algebras and matrix groups, but everything makes sense in the abstract setting.

We shall define a map

$$
\Omega^{\text {odd }}(M \times I, \mathfrak{g}) \rightarrow \Omega^{\text {even }}(M, G)
$$

from odd $\mathfrak{g}$-valued forms on $M \times I$ to even $G$-valued forms on $M$, corresponding to the projection $p: M \times I \rightarrow M$. Here a rigorous understanding of an 'even form with values in some (super)manifold', say $N$ (in our case it is a supergroup $G$ ), is a map of supermanifolds $\Pi T M \rightarrow N$ (in the case under consideration, a map $\Pi T M \rightarrow G)$.

For a matrix (super)group $G$, a $G$-valued form $g=g(x, d x)$ on an ordinary manifold is an expansion $g=g_{0}(x)+d x^{a} g_{a}(x)+\ldots+$, where the zero-order term is an invertible matrix-function such that $g_{0}(x)$ belongs to $G$ for each $x$, and the other terms are 'higher corrections'. Note, however, that the whole sum $g(x, d x)$, not only $g_{0}(x)$, has to satisfy the equations specifying the group manifold $G$, so there are relations for the higher terms as well. The whole sum must be, of course, even in the sense of total parity.

The desired map will be called the multiplicative direct image or multiplicative fiber integral and be denoted

$$
\mathrm{T} \exp p_{*}: \Omega^{\text {odd }}(M \times I, \mathfrak{g}) \rightarrow \Omega^{\text {even }}(M, G) .
$$

As the name suggests, the construction of the multiplicative direct image proceeds as follows. For a given $\omega \in \Omega^{\text {odd }}(M \times I, \mathfrak{g})$, we consider the decomposition

$$
\omega=\omega_{0}+d t \omega_{1}
$$

where $t$ is the coordinate on $I$. The form $\omega_{1}$ is an even $\mathfrak{g}$-valued form on $M$ depending on $t$ as a parameter, or, alternatively, it can be regarded as a function of $t \in I$ with values in the Lie algebra (not superalgebra!) $\Omega^{\text {even }}(M, \mathfrak{g})$. It makes sense to consider the Cauchy problem

$$
\left\{\begin{aligned}
g(0) & =1, \\
\frac{d g(t)}{d t} & =-\omega_{1} g(t)
\end{aligned}\right.
$$

(the choice of the minus sign is dictated by the geometric tradition). The solution $g(t)$, which takes values in the group $\Omega^{\text {even }}(M, G)$, will be denoted

$$
\mathrm{T} \exp \int_{0}^{t}(-\omega):=g(t)
$$

for $t \in[0,1]$. We may also need the solution at time $t=t_{1}$ of the Cauchy problem with the initial value 1 at $t=t_{0}$, which will be denoted $g\left(t_{1}, t_{0}\right)$ :

$$
g\left(t_{1}, t_{0}\right)=\mathrm{T} \exp \int_{t_{0}}^{t_{1}}(-\omega)
$$


for $t_{0}, t_{1} \in[0,1]$, so that $g(t)=g(t, 0)$. In particular, we define

$$
\omega \mapsto \mathrm{T} \exp p_{*} \omega:=g(1,0)=\mathrm{T} \exp \int_{0}^{1}(-\omega) \in \Omega^{\mathrm{even}}(M, G)
$$

to be the desired map (2.1).

Remark 2.2. The multiplicative integrals above are standard multiplicative integrals (cf. [3, 7]), and if desired they can be expressed as limits of products of exponentials or as a series of integrals of time-ordered products. We use the "T exp $\int$ " notation from among the variety of existing notation. In our case the integrands are 1-forms on $I$ with values in a Lie algebra. The corresponding Lie group $\Omega^{\text {even }}(M, G)$ is the group of the $\Omega(M)$-points of the Lie supergroup $G$ (that is, maps ПTM $\rightarrow G$ ). More detailed notation such as

$$
\mathrm{T} \exp \int_{t_{0}}^{t_{1}} d t\left(-\omega_{1}(x, d x, t)\right) \quad \text { or } \quad \mathrm{T} \exp \int_{t_{0}}^{t_{1}} D(t, d t)(-\omega(x, d x, t, d t))
$$

is also possible. In the latter formula $D(t, d t)$ stands for the Berezin integration element w.r.t. the variables $t, d t$, and the role of the Berezin integration over the odd variable $d t$ is in isolating the term $\omega_{1}$.

For an Abelian $\mathfrak{g}$, the multiplicative direct image $\mathrm{T} \exp p_{*}$ is the composition of the ordinary direct image $p_{*}=\int_{0}^{1}$ taking forms on $M \times I$ to forms on $M$ (with the same values) and the exponential map.

We shall now establish an analog of the fiberwise Stokes formula for the ordinary direct image

$$
d \circ p_{*}+p_{*} \circ d=p_{*}^{\prime} .
$$

Here $p_{*}^{\prime}$ stands for the 'integral over the fiberwise boundary', so that, for a form $\sigma(x, d x, t, d t)$ on $M \times I, p_{*}^{\prime} \sigma=\left.\sigma(x, d x, t, 0)\right|_{t=0} ^{t=1}$.

For such an analog, the first term in equation (2.6) should be replaced by the multiplicative direct image followed by the Darboux differential $g \mapsto \Delta(g)=-d g g^{-1}$, while the operator $d$ in the second term should be replaced by the non-linear operation of 'taking curvature':

$$
\omega \mapsto \operatorname{curv} \omega:=d \omega+\omega^{2} .
$$

As we shall see, certain other modifications arise for both sides and the formula as a whole is slightly more complicated than a naive analog of (2.6). (At the same time, in hindsight all extra complications allow a geometric interpretation and are geometrically natural.)

Theorem 2.1. Let $\omega=\omega_{0}+d t \omega_{1} \in \Omega^{\text {odd }}(M \times I, \mathfrak{g})$ be an odd $\mathfrak{g}$-valued form on the direct product $M \times I$. Define $g\left(t_{1}, t_{0}\right)$ as above. The following commutation formula holds:

$$
\Delta \circ \mathrm{T} \exp p_{*}+p_{*} \circ \operatorname{Ad} g(1, t) \circ \mathbf{c u r v}=p_{*}^{\prime} \circ \operatorname{Ad} g(1, t),
$$

where $p_{*}^{\prime}=\left.\right|_{t=0, d t=0} ^{t=1, d t=0}$ is the 'integral over the fiberwise boundary'.

If we denote $g:=\mathrm{T} \exp p_{*} \omega=g(1,0)$ and $\Omega:=\operatorname{curv} \omega$, then the formula reads

$$
-d g g^{-1}+\int_{0}^{1} g(1, t) \Omega g(1, t)^{-1}=\left.\omega_{0}\right|_{t=1}-g\left(\left.\omega_{0}\right|_{t=0}\right) g^{-1} .
$$


Here $p_{*}=\int_{0}^{1}$ in the second term is the ordinary fiberwise integral applied to a $\mathfrak{g}$-valued form on $M \times I$.

Remark 2.3. The form $\omega$ is odd; obviously, the $G$-valued form $g$ is even; hence the form $-d g g^{-1}$ is odd. The form $d \omega+\omega^{2}$ is even, and the fiberwise integration makes an even form odd. The term $\omega_{0}$ is odd. Therefore the main equation (2.9) is an equality between odd $\mathfrak{g}$-valued forms on $M$.

Remark 2.4. The appearance of the conjugation with $g(1, t)$ or $g=g(1,0)$ in (2.8) and (2.9) becomes geometrically transparent if one thinks of $g\left(t_{1}, t_{0}\right)$ as a 'parallel transport' along $\{x\} \times I$ from $t=t_{0}$ to $t=t_{1}$ (see more in Section 5). The whole formula (2.9) may be viewed as written at the time $t=1$.

Proof of Theorem 2.1, Equation (2.9) is an explication of (2.8), so we need to prove (2.9). We shall deduce a formula equivalent to (2.9), from which (2.9) will follow by conjugation:

$$
-g^{-1} d g+\int_{0}^{1} g(0, t)^{-1} \omega g(0, t)=g^{-1}\left(\left.\omega_{0}\right|_{t=1}\right) g-\left.\omega_{0}\right|_{t=0} .
$$

For brevity we shall use the notation $g_{t}=g(0, t)$. Consider the first term in (2.10). To obtain an expression for it, notice that $g^{-1} d g=\left.\left(g_{t}^{-1} d_{x} g_{t}\right)\right|_{t=1}$ and $\left.d_{x} g_{t}\right|_{t=0}=0$. By differentiating and using (2.2) we arrive after a simplification at

$$
\frac{d}{d t}\left(g_{t}^{-1} d_{x} g_{t}\right)=-g_{t}^{-1} d_{x} \omega_{1} g_{t}
$$

Therefore

$$
g^{-1} d g=\int_{0}^{1} d t \frac{d}{d t}\left(g_{t}^{-1} d_{x} g_{t}\right)=\int_{0}^{1} d t\left(-g_{t}^{-1} d_{x} \omega_{1} g_{t}\right) .
$$

Consider now the curvature $\omega=d \omega+\omega^{2}$. We have

$$
d \omega+\omega^{2}=d_{x} \omega_{0}+\omega_{0}^{2}+d t\left(-d_{x} \omega_{1}+\dot{\omega}_{0}+\left[\omega_{1}, \omega_{0}\right]\right),
$$

by a direct calculation (where the dot denotes the time derivative). Therefore

$$
\int_{0}^{1} g_{t}^{-1} \omega g_{t}=\int_{0}^{1} d t\left(-g_{t}^{-1} d_{x} \omega_{1} g_{t}\right)+\int_{0}^{1} d t\left(g_{t}^{-1}\left(\dot{\omega}_{0}+\left[\omega_{1}, \omega_{0}\right]\right) g_{t}\right) .
$$

It follows by combining (2.11) and (2.13) that

$$
-g^{-1} d g+\int_{0}^{1} g_{t}^{-1} \omega g_{t}=\int_{0}^{1} d t\left(g_{t}^{-1}\left(\dot{\omega}_{0}+\left[\omega_{1}, \omega_{0}\right]\right) g_{t}\right) .
$$

It remains to identify the r.h.s. of (2.14). To this end, consider $g_{t}^{-1} \omega_{0} g_{t}$. By differentiating and taking into account (2.2) we obtain, after a simplification, that

$$
\frac{d}{d t}\left(g_{t}^{-1} \omega_{0} g_{t}\right)=g_{t}^{-1}\left(\dot{\omega}_{0}+\left[\omega_{1}, \omega_{0}\right]\right) g_{t} .
$$

This is exactly what we are looking for. Now combining (2.14) with (2.15) we arrive at

$$
-g^{-1} d g+\int_{0}^{1} g_{t}^{-1} \omega g_{t}=\int_{0}^{1} d t \frac{d}{d t}\left(g_{t}^{-1} \omega_{0} g_{t}\right)
$$

which gives (2.10) as desired, since $g_{1}=g$ and $g_{0}=1$. To get (2.9) we apply the conjugation by the element $g=g(1,0)$, and the theorem is proved. 
Theorem 2.1 implies a non-Abelian analog of the familiar chain homotopy formula for ordinary differential forms. Suppose $F: M \times I \rightarrow N$ is a homotopy between maps of (super)manifolds $f_{0}, f_{1}: M \rightarrow N$ so that $F(x, t)=f_{t}(x)$ for $t=0,1$. Consider an odd form $\omega \in \Omega^{\text {odd }}(N, \mathfrak{g})$ and take its pull-back $F^{*} \omega \in \Omega^{\text {odd }}(M \times I, \mathfrak{g})$. By applying equation (2.9) to $F^{*} \omega$ and noting that taking curvature commutes with pull-backs, we arrive at the following statement.

Corollary 2.1 (Non-Abelian algebraic homotopy). The pull-backs along homotopic maps $f_{0}, f_{1}: M \rightarrow N$ of an odd $\mathfrak{g}$-valued form $\omega \in \Omega^{\text {odd }}(N, \mathfrak{g})$ are related by the formula

$$
f_{1}^{*} \omega-g\left(f_{0}^{*} \omega\right) g^{-1}=-d g g^{-1}+\int_{0}^{1} g(1, t) F^{*}(\operatorname{curv} \omega) g(1, t)^{-1},
$$

where $g=g(1,0)$ and

$$
g\left(t_{1}, t_{0}\right)=\mathrm{T} \exp \int_{t_{0}}^{t_{1}}\left(-F^{*} \omega\right) .
$$

Here $F$ is a given homotopy, $F(x, t)=f_{t}(x)$.

In particular, for flat forms, the pull-backs along homotopic maps are gaugeequivalent:

$$
f_{1}^{*} \omega=g\left(f_{0}^{*} \omega\right) g^{-1}-d g g^{-1},
$$

where 'flat' means vanishing curvature: $\operatorname{curv} \omega=d \omega+\omega^{2}=0$.

Remark 2.5. Constructions of this section can be done in a more general abstract setup. Namely, we can replace $\Omega(M, \mathfrak{g})$ by an abstract differential Lie superalgebra. Suppose $\mathfrak{g}$ (we use boldface) is such a differential Lie superalgebra (shortly, a 'dLie algebra'). Then the even forms on $M$ with values in $G$ are replaced, respectively, by points of a corresponding 'differential Lie supergroup' ('dLie group' or ' $Q$-group') $\boldsymbol{G}$. Odd forms on the cylinder $M \times I$ are modeled, in such an abstract setting, by the elements of the tensor product $\Pi \mathfrak{g} \otimes \Omega(I)=\Omega(I, \Pi \mathfrak{g})$. The construction of the multiplicative direct image and the analog of Theorem 2.1 carry through without any substantial change. The multiplicative direct image should be regarded as a supermanifold map $\Omega(I, \Pi \mathfrak{g}) \rightarrow \boldsymbol{G}$. Since our motivation is mainly differentialgeometric, we stick to the concrete setting of differential forms. However, the abstract formulation may be of independent interest. We briefly discuss it in the last section.

\section{Non-ABElian Poincaré Lemma}

Results of the previous section can be applied for obtaining a non-Abelian version of the Poincaré lemma. We can use Corollary 2.1 or argue directly, as follows. Let $\omega$ be an odd $\mathfrak{g}$-valued form on a contractible supermanifold, for example, on a star-shaped domain $U \subset \mathbb{R}^{n \mid m}$. Suppose it satisfies the Maurer-Cartan equation:

$$
d \omega+\omega^{2}=0 .
$$

Consider a contracting homotopy $H: M \times I \rightarrow M$, for example, the map $H: U \times$ $I \rightarrow U$ sending $(x, t)$ to $t x$. Take the pull-back $H^{*} \omega$ of $\omega$ and apply to it the main formula (2.9). We arrive at

$$
-d g g^{-1}+0=\left.\left(H^{*} \omega\right)\right|_{t=1, d t=0}-g\left(\left.\left(H^{*} \omega\right)\right|_{t=0, d t=0}\right) g^{-1},
$$


where

$$
g=\mathrm{T} \exp \int_{0}^{1}\left(-H^{*} \omega\right)
$$

Noticing that $\left.\left(H^{*} \omega\right)\right|_{t=1, d t=0}=\omega$ and $\left.\left(H^{*} \omega\right)\right|_{t=0, d t=0}=i^{*} \omega$, where $i$ is the inclusion of the base point to $M$, we get simply

$$
-d g g^{-1}=\omega-g\left(i^{*} \omega\right) g^{-1} .
$$

Here $i^{*} \omega$ is just an odd element of the Lie superalgebra $\mathfrak{g}$.

We have proved the following statement.

Theorem 3.1 (Non-Abelian Poincaré Lemma). On a contractible supermanifold, an odd $\mathfrak{g}$-valued form $\omega \in \Omega^{\mathrm{odd}}(M, \mathfrak{g})$ satisfying the Maurer-Cartan equation (3.1) is gauge-equivalent to a constant:

$$
\omega=g C g^{-1}-d g g^{-1},
$$

where $C \in \mathfrak{g}_{\overline{1}}$ is an odd element of the Lie superalgebra $\mathfrak{g}$ and a 'multiplicative primitive' $g \in \Omega^{\text {even }}(M, G)$ of the form $\omega$ is given by (3.2).

In particular, the formula for a 'multiplicative primitive' of an odd form satisfying the Maurer-Cartan equation on a star-shaped domain of $\mathbb{R}^{n \mid m}$ is

$$
g=\mathrm{T} \exp \int_{0}^{1}(-D(t, d t) \omega(t x, d t x+t d x))=\mathrm{T} \exp \int_{0}^{1}\left(-d t x^{a} \frac{\partial \omega}{\partial d x^{a}}(t x, t d x)\right)
$$

very similar to the classical formula for a primitive of a closed form on $\mathbb{R}^{n}$ or $\mathbb{R}^{n \mid m}$.

Since gauge transformations preserve the Maurer-Cartan equation, the constant element $C \in \mathfrak{g}_{\overline{1}}$ must also satisfy it. Hence the following holds.

Proposition 3.1. The constant $C$ (an odd element of the Lie superalgebra $\mathfrak{g}$ ) in (3.3) satisfies

$$
C^{2}=0 \quad(\text { or }[C, C]=0) ;
$$

i.e., $C$ is a homological element of the Lie superalgebra $\mathfrak{g}$.

If we split $\omega$ and other forms as $\omega=\omega_{0}+\omega_{+}$, where $\omega_{0}=\left.\omega\right|_{M}$ or more precisely $\omega_{0}=\pi^{*} i^{*} \omega=$ (here $\pi: \Pi T M \rightarrow M$ is the projection and $i: M \rightarrow \Pi T M$ is the zero section), then equation (3.3) becomes

$$
\begin{aligned}
& \omega_{0}=g_{0} C g_{0}^{-1}, \\
& \omega_{+}=\left(g C g^{-1}\right)_{+}-d g g^{-1} .
\end{aligned}
$$

In general, $\omega_{0}$ need not be constant; equation (3.6) implies $\omega_{0}$ being 'covariantly constant' w.r.t. to a flat connection: $d \omega_{0}+\left[\theta, \omega_{0}\right]=0$, where $\theta=-d g_{0} g_{0}^{-1}$.

The constant $C$ is not unique. Our proof of Theorem 3.1 gives $C$ as the value of $\omega$ at the base point $x_{0}$ of a contractible manifold $M$. In fact, it can be replaced by any constant conjugate to the value of $\omega$ at $x_{0}$ w.r.t. the adjoint action of $G$. It is easy to deduce the following statement.

Corollary 3.1. On a contractible manifold, two odd forms satisfying the MaurerCartan equation are gauge-equivalent if and only if their values at the base point are conjugate. 
Hence there is a one-to-one correspondence between the gauge equivalence classes of 'flat forms' (or flat $G$-superconnections) on a contractible manifold and the $G$ adjoint orbits of the homological elements in $\mathfrak{g}$. More properly, this should be stated not as a bijection of sets but as an isomorphism of the corresponding functors so as to allow arbitrary families.

It is possible to have non-trivial gauge equivalences between different constants, as well as self-equivalences. So the multiplicative primitive $g$ in (3.3) is not defined uniquely even for a fixed constant $C$. If

$$
\omega=h C^{\prime} h^{-1}-d h h^{-1},
$$

for some other $C^{\prime} \in \mathfrak{g}_{\overline{1}}$ and $h \in \Omega^{\text {even }}(M, G)$, then there is a relation

$$
C=k C^{\prime} k^{-1}-d k k^{-1}
$$

where $k=g^{-1} h$. The form $k \in \Omega^{\text {even }}(M, G)$ satisfies

$$
\left[C, d k k^{-1}\right]+\left(d k k^{-1}\right)^{2}=0 .
$$

Suppose there is a gauge self-equivalence

$$
C=g C g^{-1}-d g g^{-1}
$$

for a homological element $C$. Equation (3.11) can be re-written as

$$
d g=-C g+g C,
$$

with the clear intuitive meaning of the 'frame' $g$ being parallel w.r.t. the (constant) 'flat connection' $C$.

In the classical (Abelian) situation, the usual formulation of the Poincaré lemma ("every closed form is exact") is for forms of fixed positive degree. Forms of degree zero and constants appear as an exception unless inhomogeneous forms are treated. In the non-Abelian case, inhomogeneous $\mathfrak{g}$-valued forms do appear naturally. As soon as we consider them, we are forced to consider constants arising in (3.3). These constants play an important role in examples, as we shall see now.

\section{EXAMPLES}

In this section we change our notation and denote by $L$ the Lie superalgebra denoted previously by $\mathfrak{g}$. (We shall need $\mathfrak{g}$ for a different object.)

In the following examples, the Lie superalgebra $L$ will be the algebra of vector fields $\mathfrak{X}(N)$ on a (super)manifold $N$. In particular, $N$ can be a vector space. Then the Lie superalgebra $L=\mathfrak{X}(N)$ carries an extra $\mathbb{Z}$-grading corresponding to the degree in the linear coordinates on $N$. It should not be confused with parity. More generally, $N$ can be an arbitrary graded manifold, i.e., a supermanifold with an extra $\mathbb{Z}$-grading in the structure sheaf, independent of parity in general 15. We refer to such a $\mathbb{Z}$-grading as weight and denote it by $\boldsymbol{w}$.

All the considerations in the previous sections remain valid in the graded case.

Indeed, suppose that the Lie superalgebra $L$ is $\mathbb{Z}$-graded. The total weight of the elements of $\Omega(M, L)$ is the sum of ordinary degree of forms on $M$ and weight on $L$. For the operator $d+\omega$ to be homogeneous, we assume that the weight $\boldsymbol{w}(\omega)$ of the form $\omega$ equals +1 (this does not mean that $\omega$ is a 1 -form!). Recalling the construction of the multiplicative direct image on $M \times I$ for $\omega=\omega_{0}+d t \omega_{1}$ in Section 2, we see that $\boldsymbol{w}\left(\omega_{1}\right)=0$. Hence the multiplicative integral there and all the gauge transformations defined with its help have weight zero; i.e., they all preserve weights. 
Now let us turn to examples. We use the notions of Lie algebroid theory, for which the standard and encyclopedic source is Mackenzie's book [6] (see also [8]).

Example 4.1. Consider the Atiyah algebroid of a principal $G$-bundle $P \rightarrow M$, which is a transitive Lie algebroid over $M$. See [6]. (Here $G$ is a Lie group, nothing to do with what it was in the previous sections.) It is defined as $T P / G$, and it inherits the structure of a vector bundle over $M$. There is an epimorphism of vector bundles $T P / G \rightarrow T M$, which is the anchor in the Lie algebroid structure. Let us reverse parity in the fibers and consider the supermanifold $\Pi T P / G$. We can consider it as a fiber bundle over $\Pi T M$,

$$
\Pi T P / G \rightarrow \Pi T M .
$$

The standard fiber of (4.1) can be identified with $\Pi \mathfrak{g}$, where $\mathfrak{g}$ is the Lie algebra of $G$. The transition functions have the form

$$
\xi_{\alpha}=g_{\alpha \beta} \xi_{\beta} g_{\alpha \beta}^{-1}-d g_{\alpha \beta} g_{\alpha \beta}^{-1}
$$

if $g_{\alpha \beta}$ is the cocycle defining the principal bundle $P \rightarrow M$. They are linear in $\xi, d x$, but affine in $\xi$ alone. Here $\xi$, or $\xi_{\alpha}$ in a particular local trivialization, belongs to $\Pi \mathfrak{g}$. Hence (4.1) is an affine bundle over ПTM. The Lie algebroid structure of the Atiyah algebroid is encoded in the following homological vector field on $\Pi T P / G$ :

$$
Q=d x^{a} \frac{\partial}{\partial x^{a}}+\frac{1}{2} \xi^{i} \xi^{j} C_{j i}^{k} \frac{\partial}{\partial \xi^{k}} .
$$

Here $x^{a}$ are local coordinates on $M$ and $\xi^{i}$ are linear coordinates on $\Pi \mathfrak{g}$. The tensor $C_{i j}^{k}$ gives the structure constants of the Lie algebra $\mathfrak{g}$. One may consider the second term in (4.3) as a constant element $C$ of the Lie superalgebra $L=\mathfrak{X}(\Pi \mathfrak{g})$ of the vector fields on the supermanifold $\Pi \mathfrak{g}$, so $Q$ has the appearance

$$
Q=d+C, \quad \text { where } \quad C \in \mathfrak{X}(\Pi \mathfrak{g}) .
$$

Note that $C$ is odd and has weight 1 . It satisfies $C^{2}=0$. In a different language, $C$ is the differential of the standard cochain complex of $\mathfrak{g}$ usually denoted as $\delta$ (the Chevalley-Eilenberg or Cartan differential). It is a remarkable fact, directly verifiable, that the decomposition (4.4) survives transformations of the form (4.2).

Example 4.2. Let $E \rightarrow M$ now be an arbitrary transitive Lie algebroid; see 6]. The anchor map $E \rightarrow T M$ is epimorphic, so we can again consider it as a fiber bundle. One can check that it is an affine bundle. Consider the vector bundles over $M$ with reversed parity in the fibers. We have the affine bundle

$$
\Pi E \rightarrow \Pi T M .
$$

If we denote coordinates in the fiber of (4.5) by $\xi^{i}$ and local coordinates on $M$ by $x^{a}$ as above, so that on $\Pi T M$ the coordinates are $x^{a}, d x^{a}, \xi^{i}$, the changes of coordinates are

$$
\left\{\begin{aligned}
x^{a} & =x^{a}\left(x^{\prime}\right) \\
d x^{a} & =d x^{a^{\prime}} \frac{\partial x^{a}}{\partial x^{a^{\prime}}} \\
\xi^{i} & =d x^{a^{\prime}} T_{a^{\prime}}^{i}\left(x^{\prime}\right)+\xi^{i^{\prime}} T_{i^{\prime}}^{i}\left(x^{\prime}\right),
\end{aligned}\right.
$$


with some matrices $T_{a^{\prime}}^{i}\left(x^{\prime}\right)$ and $T_{i^{\prime}}^{i}\left(x^{\prime}\right)$. The homological vector field $Q$ on $\Pi E$ defining the structure of a Lie algebroid over $M$ has the form

$$
Q=d+\frac{1}{2}\left(\xi^{i} \xi^{j} Q_{j i}^{k}(x)+2 \xi^{i} d x^{a} Q_{a i}^{k}(x)+d x^{a} d x^{b} Q_{b a}^{k}(x)\right) \frac{\partial}{\partial \xi^{k}}
$$

in local coordinates, with $d=d x^{a} \partial / \partial x^{a}$, or

$$
Q=d+\omega
$$

where

$$
\omega=\frac{1}{2}\left(\xi^{i} \xi^{j} Q_{j i}^{k}(x)+2 \xi^{i} d x^{a} Q_{a i}^{k}(x)+d x^{a} d x^{b} Q_{b a}^{k}(x)\right) \frac{\partial}{\partial \xi^{k}}
$$

can be regarded as a (local) form on $M$ with values in the Lie superalgebra $L=$ $\mathfrak{X}(\Pi V)$. Here $V$ is the standard fiber of $E \rightarrow T M$. Note that the form $\omega$ is odd and inhomogeneous w.r.t. ordinary degree. However, it is homogeneous of weight +1 if weight is counted as the sum of form degree and grading of vector fields on $\Pi V$. Claim: the form (4.9) is gauge-equivalent to a constant. This amounts to saying that by an appropriate affine transformation of the coordinates $\xi$,

$$
\xi=\eta \cdot A(x)+\beta,
$$

where the matrix $A(x)$ depends only on $x$ and $\beta$ is a 1 -form, one can transform the vector field $Q$ on $\Pi E$ to the form

$$
Q=d+C, \quad \text { where } \quad C=\frac{1}{2} \eta^{i} \eta^{j} C_{j i}^{k} \frac{\partial}{\partial \eta^{k}} \in \mathfrak{X}(\Pi V),
$$

with constant $C_{i j}^{k}$. This follows by a direct application of Theorem 3.1. That the transformation is affine follows from the preservation of weights and can be seen from an explicit construction of the multiplicative integrals. In particular, $C^{2}=0$; therefore the vector space $V$ is endowed with a structure of a Lie algebra. This encapsulates a substantial part of the transitive Lie algebroid theory [6, Ch. 8].

The previous example prompts an immediate analogy. A few words should be said before we pass to it.

Recall that a $Q$-manifold is an arbitrary supermanifold endowed with a homological vector field. $Q$-manifolds should be regarded, together with Poisson manifolds and Schouten (odd Poisson) manifolds, as one of the three equally important nonlinear generalizations of Lie algebras 1 Vaintrob noted [14 that a Lie algebroid structure on a vector bundle $E$ is very efficiently described by a homological vector field on the parity-reversed total space $\Pi E$. This approach was further developed in particular in [10, [15], 11, and [16]. This is what we used above. (We use the notation of [15].) For a Lie algebroid the corresponding field $Q$ has weight +1 w.r.t. the linear coordinates in the fibers. Dropping this restriction leads to objects such as strongly homotopy Lie (or $L_{\infty}$ ) algebras and algebroids. On the other hand, constructions such as the "cotangent construction of the Drinfeld double' for Lie bialgebroids [10] (compare also [5]) take us out of the world of vector bundles. Prompted by that, it is proper to replace a lacking linear structure by weight and consider graded manifolds instead of vector bundles [15. In particular,

\footnotetext{
${ }^{1}$ A Lie algebra multiplication of the elements of $\mathfrak{g}$ has precisely three geometric manifestations: as a linear Poisson bracket on $\mathfrak{g}^{*}$, as a linear Schouten bracket on $\Pi \mathfrak{g}^{*}$, and as a quadratic homological vector field on $\Pi \mathfrak{g}$.
} 
a non-negatively graded $Q$-manifold with $\boldsymbol{w}(Q)=+1$ is the closest analog of a Lie algebroid and should be regarded as the 'non-linear version' of such.

Example 4.3 ("Non-linear transitive Lie algebroids"). Consider a fiber bundle

$$
p: E \rightarrow \Pi T M
$$

in the category of graded manifolds. Here $E$ is a non-negatively graded manifold (in particular, a supermanifold) $2 M$ is an ordinary supermanifold with trivial grading, and $\Pi T M$ is considered with the standard vector bundle grading. (More pedantically, it should be denoted by $\Pi T[1] M$.) Suppose there is a homological vector field $Q \in \mathfrak{X}(E)$ of weight +1 such that $Q$ on $E$ and $d$ on $\Pi T M$ are $p$-related. Then in suitable local coordinates $Q$ has the form

$$
Q=d x^{a} \frac{\partial}{\partial x^{a}}+Q^{i}(x, d x, y) \frac{\partial}{\partial y^{i}},
$$

where $x^{a}$ are local coordinates on $M, d x^{a}$ are their differentials, and $y^{i}$ are coordinates in the fiber over $\Pi T M$. The changes of coordinates on $E$ have the form

$$
\left\{\begin{aligned}
x^{a} & =x^{a}\left(x^{\prime}\right), \\
d x^{a} & =d x^{a^{\prime}} \frac{\partial x^{a}}{\partial x^{a^{\prime}}}, \\
y^{i} & =y^{i}\left(x^{\prime}, d x^{\prime}, y^{\prime}\right) .
\end{aligned}\right.
$$

The appearance of $d$ as the first term in (4.12) follows from the condition that $Q$ and $d$ are $p$-related. We have $\boldsymbol{w}\left(x^{a}\right)=0, \boldsymbol{w}\left(d x^{a}\right)=1$, and $\boldsymbol{w}\left(y^{i}\right)=w^{i}>0$ are some positive integers. The coordinate transformations (4.13) are homogeneous polynomials in $d x^{a^{\prime}}, y^{i^{\prime}}$. The coefficients $Q^{i}(x, d x, y)$ in (4.12) have weights $w^{i}+1$ respectively. Compare with (4.7). The entire structure consisting of the fiber bundle $E \rightarrow \Pi T M$ and the field $Q$ with these properties should be regarded as a transitive non-linear Lie algebroid. The bundle projection $p: E \rightarrow \Pi T M$ plays the role of the anchor. A construction of this type with an extra restriction - see below - was put forward in 13, 4. They introduced $Q$-bundles $p: E \rightarrow B$, where both $E$ and $B$ are $Q$-manifolds and the projection is a $Q$-morphism (the respective vector fields are $p$-related) with a condition of 'local triviality' in the following strong sense. The standard fiber $F$ is a $Q$-manifold, and there is a bundle atlas where in local trivializations the homological vector field $Q_{E}$ on $E$ takes the form of the sum

$$
Q_{E}=Q_{B}+Q_{F},
$$

where $Q_{B}$ and $Q_{F}$ are the homological vector fields on $B$ and $F$; the transition functions are such that they respect the decomposition (4.14) ${ }^{3}$ The main case is when the $Q$-manifold $B$ equals $\Pi T M$ for some $M$. Therefore, it is a transitive non-linear Lie algebroid in our sense subject to the condition of the existence of a bundle atlas for $E \rightarrow \Pi T M$ with the described properties. We shall refer to this, in particular to equation (4.14), as a Kotov-Strobl gauge. Our Theorem 3.1 therefore implies that every transitive non-linear Lie algebroid admits a KotovStrobl gauge. This means that it is possible to transform coordinates $y^{i}$ to $z^{j}$ by

\footnotetext{
${ }^{2}$ Comparing to the previous example, $E$ now stands for what was $\Pi E$.

${ }^{3}$ There are more conditions to be mentioned: the total space and the base are graded manifolds, and the homological fields have weights +1 (in fact, in [13, 4] it is assumed that grading induces parity, which is absolutely unnecessary), and some Lie group of transformations preserving (4.14) is fixed as a part of data.
} 
a homogeneous transformation depending on $x^{a}, d x^{a}$ as parameters in each bundle chart so that the vector field (4.12) becomes

$$
Q=d x^{a} \frac{\partial}{\partial x^{a}}+Q^{i}(z) \frac{\partial}{\partial z^{i}}
$$

(no dependence on $x^{a}, d x^{a}$ in the second term). The graded Lie superalgebra $L$ here is the algebra of all graded vector fields $\mathfrak{X}(F)$ on the standard fiber $F$. In particular, we conclude that constructions of [4] are valid for arbitrary transitive non-linear Lie algebroids, without extra restrictions.

An appearance of fiber bundles of the form $E \rightarrow \Pi T M$ may look artificial. It helps to put it into a broader perspective as follows. Consider an arbitrary nonnegatively graded $Q$-manifold $E$ where $\boldsymbol{w}(Q)=+1$. As noted before, it should be regarded as a general non-linear Lie algebroid. At first glance, there is no fiber bundle there. Recall, however, that any non-negatively graded manifold $E$ gives rise to a finite tower of fibrations 15

$$
E=E_{N} \rightarrow E_{N-1} \rightarrow \ldots \rightarrow E_{2} \rightarrow E_{1} \rightarrow E_{0},
$$

where functions on $E_{0}$ have weight $0, E_{1} \rightarrow E_{0}$ is a vector bundle, and $E_{k+1} \rightarrow E_{k}$ for higher $k$ are affine bundles. This can be assembled into a single fiber bundle

$$
E \rightarrow M
$$

where $M=E_{0}$, the coordinates on the standard fiber have positive weights and the transition functions are homogeneous polynomials. The supermanifold $M$ is embedded into $E$ as a 'zero section'. The homological vector field $Q \in \mathfrak{X}(E)$ in bundle coordinates has the form

$$
Q=Q^{a}(x, y) \frac{\partial}{\partial x^{a}}+Q^{i}(x, y) \frac{\partial}{\partial y^{i}},
$$

where $x^{a}$ are local coordinates on the base $M$ and $y^{i}$ are coordinates on the fiber. We define a map

$$
a: E \rightarrow \Pi T M
$$

by the formula $a^{*}\left(x^{a}\right)=x^{a}, a^{*}\left(d x^{a}\right)=Q^{a}(x, y)$ and call it the anchor for $E$. (Welldefined because we have a bundle, so the transformation of the coordinates is of the form $x^{a}=x^{a}\left(x^{\prime}\right), y^{i}=y^{i}\left(x^{\prime}, y^{\prime}\right)$.) It is a bundle map over $M$ and a $Q$-morphism (by a direct check using $Q^{2}=0$ ). In the case of ordinary Lie algebroids it coincides with the usual anchor after the parity reversion. If the anchor $E \rightarrow \Pi T M$ is a surjective submersion, we call the non-linear Lie algebroid $E$ transitive, as in the ordinary case [6], and so we arrive at the setup of Example 4.3 .

Remark 4.1. A tautological 'anchor' makes sense for any $Q$-manifold $M$ without assumption of grading: it is the vector field $Q$ itself considered as a map $M \rightarrow$ $\Pi T M$, which is a $Q$-morphism. Of course such an 'anchor' carries information about all the algebraic structures contained in the vector field $Q$, so the name becomes a bit arbitrary.

More about the 'non-linear Lie algebroids' and the algebraic structure arising from them can be found in our new paper [17. 


\section{Discussion}

We have studied inhomogeneous or pseudodifferential odd forms on a supermanifold $M$ taking values in a Lie superalgebra $\mathfrak{g}$ and have showed that if they satisfy the Maurer-Cartan (zero curvature) equation $d \omega+\omega^{2}=0$, then, on a contractible supermanifold, they are gauge-equivalent to constants

$$
\omega=g C g^{-1}-d g g^{-1},
$$

where $g$ is an even $G$-valued form and $C \in \mathfrak{g}_{\overline{1}}$ such that $C^{2}=0$ (Theorem 3.1). The statement follows from more general claims (Theorem 2.1] and Corollary 2.1), which form a non-Abelian analog for $\mathfrak{g}$-valued pseudodifferential forms of the standard chain homotopy construction. For applications, the presence of an extra $\mathbb{Z}$-grading different from the naive form degree may be important; all statements hold true with such an extra grading taken into account. Our statements are particularly useful in examples related to Lie algebroids and their generalizations, but there is little doubt about their broader significance.

Let us emphasize that in the existing literature, especially in physics (gauge theory), the expression "non-Abelian Poincaré lemma" has been almost exclusively understood as the familiar statement about Lie algebra-valued 1-forms. Our usage is different, and this should not lead to confusion. The principal novelty of the present work is that we study inhomogeneous (or pseudodifferential) odd forms instead of 1-forms. When the coefficients belong to a Lie superalgebra, this results in mixing terms of different form degrees. This has not been done previously, and we were naturally led to it by applications such as Lie algebroids. The appearances of an odd constant $C$ and of a gauge transformation generated by a $G$-valued form (instead of a function) are two distinctive features of our formula (5.1).

Remark 5.1. One exception to the typical usage of "non-Abelian Poincaré lemma" as the statement about 1-forms is paper [1, which is concerned with a "non-Abelian de Rham theory' regarded as the theory of certain cohomological sets constructed from matrix-valued $p$-forms. In [1, there are a '1-dimensional non-Abelian Poincaré lemma', meaning the familiar statement for 1-forms; a '2-dimensional non-Abelian Poincaré lemma', meaning a condition of local solubility of $d \theta+\theta^{2}=\Theta, \theta$ and $\Theta$ being matrix-valued 1- and 2-forms respectively; and a '3-dimensional non-Abelian Poincaré lemma', meaning a condition of local solubility of $d \Theta+[\theta, \Theta]=\Psi$ for a 3 -form $\Psi$. There is no relation between that and our work.

On the other hand, the results of the present paper are related to homotopy theory considered from the viewpoint of algebraic deformation theory.

After the first version of this paper was finished and circulated, Stasheff told us about his "secret" work with Schlessinger [12] (still an unpublished manuscript). We also learned about the preprint by Chuang and Lazarev, now published [2]. Stasheff pointed out to us that certain formulas such as our equation (2.19) in Corollary 2.1 reminded him of some statements in 12 and kindly shared the text with us. To explain the relation, it is convenient to use a reformulation of the Main Homotopy Theorem of Schlessinger-Stasheff [12] given by Chuang and Lazarev [2. The theorem concerns Maurer-Cartan elements in a nilpotent (or pro-nilpotent) $\mathrm{dg}$ Lie algebra. The statement is that any two such elements are gauge-equivalent if and only if they are homotopic (the exact notion of homotopy is explained below). Apart from considering dg algebras, not superalgebras, and imposing the 
pro-nilpotence condition, this statement is close to an abstract algebraic version of the zero curvature case of our Corollary 2.1. The method of the proof used in [2] - formal path-ordered integrals with values in a completion of the universal enveloping algebra (here the pro-nilpotence is used) - is also close to ours but is more algebraic in spirit. So in spite of a really different language, there is an overlap between some of our results and those of [12, [2]; a comparison can be presented as follows.

Because we are motivated by geometric applications, we mainly deal with differential forms, while Schlessinger-Stasheff and Chuang-Lazarev work in the abstract setting of dg Lie algebras. They consider only Maurer-Cartan elements. For us, forms satisfying the Maurer-Cartan equation are, surely, of great importance, but we start by establishing general formulas such as 'non-Abelian chain homotopy' for the arbitrary case.

We mentioned at the end of section 2 (see Remark 2.5) that results of that section extend to a more abstract framework. This was not our original goal, but may be of independent interest and allows, in particular, further comparison with [12] and [2. We shall now give more details. We shall obtain a generalization of both our Theorem 2.1 and the 'Main Homotopy Theorem' quoted above.

Consider a differential Lie superalgebra; i.e., a Lie superalgebra $\mathfrak{g}$ endowed with an odd derivation $d$ such that $d^{2}=0$. A corresponding Lie supergroup $\boldsymbol{G}$ has a $Q$-group structure; i.e., it is endowed with a homological vector field $Q$ such that

$$
Q\left(g_{1} g_{2}\right)=Q\left(g_{1}\right) g_{2}+g_{1} Q\left(g_{2}\right) .
$$

(In particular, $Q(1)=0$ and $Q\left(g^{-1}\right)=-g^{-1} Q(g) g^{-1}$.) In the main text, both $d$ and $Q$ are given by the de Rham differential. It is convenient, if no confusion is possible, to continue to use the notation $d$ for both operations in the abstract setup as well. So from now on we replace $Q$ by $d$ and write $d g$ for $d(g)$. Consider odd elements of $\mathfrak{g}$. They should be properly regarded as points of the supermanifold $\Pi \mathfrak{g}$. The notion of gauge-equivalence carries over verbatim to them:

$$
\omega^{\prime}=g \omega g^{-1}-d g g^{-1} .
$$

Here $g$ should be regarded as a point of the supergroup $\boldsymbol{G}$. The Darboux differential on $\boldsymbol{G}$ is defined as $\Delta(g)=-d g g^{-1}$. It is a supermanifold map $\boldsymbol{G} \rightarrow \Pi \mathfrak{g}$. We can speak about the curvature $\operatorname{curv} \omega=d \omega+\omega^{2}$ (where $\omega^{2}=[\omega, \omega] / 2$, as usual) of an element $\omega \in \Pi \mathfrak{g}$. (Upon natural identifications, curv $\omega$ is exactly the value of the homological field defining the dLie algebra structure of $\mathfrak{g}$ at the argument $\omega$.) Consider forms on the unit segment $I$ with values in $\mathfrak{g}$. They make a dLie algebra w.r.t. the bracket extended from $\mathfrak{g}$ by $\Omega(I)$-linearity and the operator $D$,

$$
D \omega=d \omega+d t \frac{\partial \omega}{\partial t}
$$

as the differential, where the first term is the differential on $\mathfrak{g}$ applied 'pointwise'. (This models $\mathfrak{g}$-valued forms on the cylinder $M \times I$.) We can now define the multiplicative integral

$$
g\left(t_{1}, t_{0}\right)=\mathrm{T} \exp \int_{t_{0}}^{t_{1}}(-\omega)
$$

of an odd element of $\omega(I, \mathfrak{g})$. It takes values in the supergroup $\boldsymbol{G}$. We denote by $g$ without arguments the element $g(1,0)$. 
Lemma 5.1. The following identity holds:

$$
\Delta\left(\mathrm{T} \exp \int_{0}^{1}(-\omega)\right)+\int_{0}^{1} \operatorname{Ad} g(1, t)(\operatorname{curv} \omega)=\omega_{0}(1)-\operatorname{Ad} g \omega_{0}(0)
$$

or

$$
-d g g^{-1}+\int_{0}^{1} g(1, t)(\operatorname{curv} \omega) g(1, t)^{-1}=\omega_{0}(1)-g \omega_{0}(0) g^{-1} .
$$

Proof. This is an analog of Theorem 2.1, and its proof can be repeated verbatim.

If we elaborate the curvature appearing under the integral above, we obtain the following. For an odd $\omega=\omega_{0}+d t \omega_{1}$,

$$
\begin{aligned}
\operatorname{curv} \omega & =d \omega_{0}+\omega_{0}^{2}+d t\left(-d \omega_{1}+\frac{\partial \omega_{0}}{\partial t}-\left[\omega_{0}, \omega_{1}\right]\right) \\
& =\operatorname{curv} \omega_{0}+d t\left(-d \omega_{1}+\frac{\partial \omega_{0}}{\partial t}-\left[\omega_{0}, \omega_{1}\right]\right)
\end{aligned}
$$

(compare equation (2.12)). Clearly, the input to the integral is given only by the second term (that contains $d t$ ). If the coefficient vanishes, the integral vanishes from the formula.

Now we can define a notion of homotopy for arbitrary odd elements of a dLie algebra $\mathfrak{g}$ that extends the notion used in [2] for the Maurer-Cartan elements.

We say that two odd elements, $\omega^{0}$ and $\omega^{1}$, in $\mathfrak{g}$ are homotopic if there is an odd element $\omega \in \omega(I, \mathfrak{g})$ such that:

1) $\omega$ specializes to $\omega^{0}$ and $\omega^{1}$ for $t=0$ and $t=1$ (and $d t=0$ ), and

2) the curvature of $\omega$ does not contain $d t$.

The element $\omega$ is called a homotopy between $\omega^{0}$ and $\omega^{1}$.

(It will be shown later that for the case of zero curvature this reproduces the notion of homotopy for the Maurer-Cartan elements.)

Example 5.1. Let $\mathfrak{g}=\Omega(M)$ for some manifold $M$, with the zero bracket and the de Rham $d$. By an easy exercise one can see that two forms $\omega^{0}, \omega^{1} \in \Omega(M)$ (parity is not essential here) are homotopic in the above sense if and only if $\omega^{1}-\omega^{0}=d \sigma$ for some $\sigma \in \Omega(M)$. So in this toy case, 'homotopy' is just a cohomology relation for not necessarily closed forms.

Now the main statement.

Theorem 5.2. Let $\mathfrak{g}$ be a dLie algebra and $\boldsymbol{G}$ be a corresponding dLie group. Let $\boldsymbol{G}$ be connected. Then two odd elements $\omega^{0}$ and $\omega^{1}$ in $\mathfrak{g}$ are homotopic if and only if they are gauge-equivalent.

Proof. Suppose $\omega^{0}$ and $\omega^{1}$ are homotopic. Then there is an odd element $\omega=$ $\omega_{0}+d t \omega_{1} \in \Omega(I, \mathfrak{g})$ such that $\omega_{0}(0)=\omega^{0}, \omega_{0}(1)=\omega^{1}$ and $\operatorname{curv} \omega$ does not contain $d t$. Then, by Lemma 5.1.

$$
-d g g^{-1}=\omega_{0}(1)-g \omega_{0}(0) g^{-1}
$$

(there is no second term on the left-hand side because the integral vanishes) or

$$
\omega^{1}=g \omega^{0} g^{-1}-d g g^{-1}
$$

i.e., $\omega^{0}$ and $\omega^{1}$ are gauge-equivalent.

Conversely, suppose $\omega^{0}$ and $\omega^{1}$ are gauge-equivalent, i.e., $\omega^{1}=g \omega^{0} g^{-1}-d g g^{-1}$, for some $g \in \boldsymbol{G}$. Because we have assumed that $\boldsymbol{G}$ is connected, we can consider a 
path $h=h(t)$ such that $h(0)=1$ and $h(1)=g$. Define $\omega=\omega_{0}+d t \omega_{1} \in \Omega(I, \mathfrak{g})$ by setting

$$
\omega_{0}:=h \omega^{0} h^{-1}-d h h^{-1}
$$

(so that we indeed have $\omega_{0}(0)=\omega^{0}$ and $\omega_{0}(1)=\omega^{1}$ ) and

$$
\omega_{1}:=-\dot{h} h^{-1} \text {. }
$$

By a direct calculation, we can see that the equation

$$
\frac{\partial \omega_{0}}{\partial t}=-d \omega_{1}+\left[\omega_{0}, \omega_{1}\right]
$$

is satisfied. Therefore $\omega$ is a desired homotopy between $\omega^{0}$ and $\omega^{1}$.

Theorem 5.2 is a generalization of the Main Homotopy Theorem quoted above, which is Theorem 4.4 in [2].

Remark 5.2. The notion of homotopy for the Maurer-Cartan elements used in [2] ('Sullivan homotopy') is as follows: two Maurer-Cartan elements $\omega^{0}$ and $\omega^{1}$ in $\mathfrak{g}$ are homotopic if there is a Maurer-Cartan element $\omega \in \omega(I, \mathfrak{g})$ specializing to $\omega^{0}$ and $\omega^{1}$ resp. for $t=0$ and $t=1$. If we apply our definition instead, it is not known a priori that a homotopy $\omega$ is Maurer-Cartan. However, it automatically is if only $\omega^{0}$ and $\omega^{1}$ are Maurer-Cartan due to the following observation. We know that curv $\omega$ not containing $d t$ translates into the equation

$$
\frac{\partial \omega_{0}}{\partial t}=-d \omega_{1}+\left[\omega_{0}, \omega_{1}\right]
$$

Consider the time derivative of $\operatorname{curv} \omega_{0}$. The above equation implies

$$
\frac{\partial \operatorname{curv} \omega_{0}}{\partial t}=-\left[\omega_{1}, \operatorname{curv} \omega_{0}\right] \text {. }
$$

This is a linear differential equation for $\operatorname{curv} \omega_{0}$. So if $\operatorname{curv} \omega_{0}(0)=0$, then $\operatorname{curv} \omega_{0}=0$ for all $t$. Since, for a homotopy $\omega, \operatorname{curv} \omega=\operatorname{curv} \omega_{0}$, we conclude that a homotopy between Maurer-Cartan elements of $\mathfrak{g}$ is necessarily given by a Maurer-Cartan element of $\Omega(I, \mathfrak{g})$, and therefore our notion of homotopy for arbitrary odd elements of a dLie algebra agrees with the notion previously used for the Maurer-Cartan elements.

Possibly one can obtain further generalizations to $L_{\infty}$-algebras and arbitrary $Q$-manifolds.

In conclusion, we wish to comment on the geometric meaning of the multiplicative integrals in this paper. (This requires a discussion because they are not integrals of 1 -forms, in general.)

For an ordinary 1-form, one can integrate it over paths, for a closed form obtaining a function of the upper limit, which is a primitive of the form. Similarly, for a 1-form $\omega \in \Omega^{1}(M, \mathfrak{g})$ with values in a Lie (super)algebra, one can take multiplicative integrals over paths, which represent the parallel transport w.r.t. the corresponding connection. In the flat case this gives a $G$-valued function $g$ on $M$ such that $\omega=\Delta(g)$. The picture seems to break down when a 1-form is replaced by a form of higher degree or, even more, by an inhomogeneous or pseudodifferential form - how can one integrate something that is not a 1-form over a path? The key, of course, is that we deal with families of paths, not individual paths. Even if what we integrate is not a 1-form, the integral over paths in a family does make sense, giving a form, rather than a function, on the space of parameters. In the flat case, 
it is just the supermanifold $M$ parameterizing the upper limit or $M \times M$ for the two limits. Therefore (3.4) and similar formulas have a geometric interpretation as the parallel transports over paths in a family with base $M$ with respect to a flat Quillen superconnection $d+\omega$, where such a parallel transport is described by a $G$-valued form on $M$.

It comes about naturally to drop the condition of flatness and consider the space of all paths on $M$ (of some appropriate class, such as, e.g., piecewise smooth). It is a "path groupoid" Path $(M)$ (objects are points of $M$, arrows are paths). More precisely, consider a principal $G$-bundle $P \rightarrow M$ over $M$, where $G$ is a supergroup 4 endowed with Quillen's superconnection $\nabla$. Then the parallel transport corresponding to $\nabla$ is an even form on the path groupoid Path $(M)$ on $M$ with values in the transitive (super) Lie groupoid $\Phi(P)$ associated with the principal bundle $P \rightarrow M$; i.e., it is a supermanifold map

$$
\tau: \Pi T \operatorname{Path}(M) \rightarrow \Phi(P),
$$

which is a morphism of Lie groupoids 5

\section{ACKNOWLEDGMENTS}

The author thanks H. M. Khudaverdian and K. C. H. Mackenzie for many inspiring discussions. He is very grateful to J. D. Stasheff for reading through the first version of the text and for providing numerous valuable comments on style as well as mathematics and for sharing the unpublished manuscript [12. The author is also grateful to A. Lazarev for attracting his attention to paper [2]. It is a pleasure to thank them all.

\section{REFERENCES}

[1] A. Asada. Nonabelian Poincaré lemma. In Differential geometry, Peñíscola 1985, volume 1209 of Lecture Notes in Math., pages 37-65. Springer, Berlin, 1986. MR863744 (88d:58005)

[2] J. Chuang and A. Lazarev. Feynman diagrams and minimal models for operadic algebras. J. London Math. Soc., 81 (2):317-337, 2010. MR2602998 (2011c:18011)

[3] F. R. Gantmacher. Applications of the theory of matrices. Translated by J. L. Brenner, with the assistance of D. W. Bushaw and S. Evanusa. Interscience Publishers, Inc., New York, 1959. MR0107648(21:6372b)

[4] A. Kotov and Th. Strobl. Characteristic classes associated to $Q$-bundles. 2007, arXiv:0711.4106v1 [math.DG].

[5] K. C. H. Mackenzie. Drinfel'd doubles and Ehresmann doubles for Lie algebroids and Lie bialgebroids. Electron. Res. Announc. Amer. Math. Soc., 4:74-87 (electronic), 1998. MR.1650045 (2000c:58035)

[6] K. C. H. Mackenzie. General theory of Lie groupoids and Lie algebroids, volume 213 of London Mathematical Society Lecture Note Series. Cambridge University Press, Cambridge, 2005. MR2157566 (2006k:58035)

[7] O. V. Manturov. A multiplicative integral. In Problems in geometry, Vol. 22 (Russian), Itogi Nauki i Tekhniki, pages 167-215, 219. Akad. Nauk SSSR Vsesoyuz. Inst. Nauchn. i Tekhn. Inform., Moscow, 1990. Translated in J. Soviet Math., 55(5): 2042-2076, 1991. MR1099222 (92f:58021)

\footnotetext{
${ }^{4}$ It may be natural to consider bundles over $\Pi T M$ from the start.

${ }^{5} \mathbf{P a t h}(M)$ is not exactly a groupoid, because even if we consider paths up to reparametrization and thus achieve the associativity, there remain problems with units and inverses. Still, (5.2) is a morphism to a genuine Lie groupoid. This leads to the temptation to formally introduce a universal groupoid with a morphism from Path $(M)$ to it as some sort of 'non-Abelian singular 1-chains'.
} 
[8] I. Moerdijk and J. Mrčun. Introduction to foliations and Lie groupoids, volume 91 of Cambridge Studies in Advanced Mathematics. Cambridge University Press, Cambridge, 2003. MR2012261 (2005c:58039)

[9] D. Quillen. Superconnections and the Chern character. Topology, 24(1):89-95, 1985. MR790678 (86m:58010)

[10] D. Roytenberg. Courant algebroids, derived brackets and even symplectic supermanifolds. Ph.D. thesis, UC Berkeley, 1999, ProQuest LLC. MR2699145

[11] D. Roytenberg. On the structure of graded symplectic supermanifolds and Courant algebroids. In Quantization, Poisson Brackets and Beyond, volume 315 of Contemp. Math., pages 169186. Amer. Math. Soc., Providence, RI, 2002. MR.1958835(2004i:53116)

[12] M. Schlessinger and J. Stasheff. Deformation theory and rational homotopy type. (Manuscript in preparation.)

[13] Th. Strobl. Talks at the conference "Algebraic Aspects in Geometry", Będlewo, Poland, 1723 October 2007, and the XXVII Workshop on Geometric Methods in Physics, Białowieża, Poland, 29 June to 5 July 2008.

[14] A. Yu. Vaŭntrob. Lie algebroids and homological vector fields. Uspekhi Matem. Nauk, 52(2):428-429, 1997. MR1480150

[15] Th. Voronov. Graded manifolds and Drinfeld doubles for Lie bialgebroids. In Quantization, Poisson Brackets and Beyond, volume 315 of Contemp. Math., pages 131-168. Amer. Math. Soc., Providence, RI, 2002. MR.1958834(2004f:53098)

[16] Th. Voronov. Mackenzie theory and $Q$-manifolds. arXiv:math/0608111v2 [math.DG].

[17] Th. Voronov. Q-manifolds and higher analogs of Lie algebroids. In XXIX Workshop on Geometric Methods in Physics. AIP CP 1307, pages 191-202. Amer. Inst. Phys., Melville, NY, 2010.

School of Mathematics, University of Manchester, Oxford Road, Manchester, M13 9PL, United KingDom

E-mail address: theodore.voronov@manchester.ac.uk 